Acta Mechanica Slovaca 16(1): 84 - 89, 2012 DOI: 10.2478/v10147-012-0010-y (0) 2012 VersitaOpen Ltd. All rights reserved.

* Corresponding autho

Phone +421556022470

E-mail address:a.tofil@pollub.p

(PhD. Ing. Arkadiusz Tofil)

Article information

Article history: AMS-Volume16-No.1-00146-12

Received 6 February 2012

Accepted 15 March 2012

\section{Efect of Hall Current and Slip Conditions on Heat and Mass Transfer of Unsteady MHD Flow of a Viscoelastic...}

\author{
J.I. Oahimire ${ }^{1 *}$, B. I Olajuwon ${ }^{2}$ \\ 'Department of Mathematics, University of Port Harcourt, Port Harcourt, Nigeria. \\ ${ }^{2}$ Department of Mathematics, Federal University of Agriculture, Abeokuta, Nigeria.
}

\section{KEY WORDS}

Micropolar fluid, perturbation technique,heat and mass transfer, Hall effect and slip conditions, porous medium...

\begin{abstract}
Heat and mass transfer effects on unsteady flow of a viscoelastic micropolar fluid over an infinite moving permeable plate in a saturated porous medium in the presence of a transverse magnetic field with Hall effect and slip conditions are studied.The governing system of partial differential equations is transformed to dimensionless equations using dimensionless variables. The dimensionless equations are then solved analytically using perturbation technique to obtain the expression for velocity, microrotation, temperature and concentration. With the help of graphs, the effects of the various important parameters entering into the problem on the velocity, microrotation, temperature and concentration fields within the boundary layer are discussed. Also the effects of the pertinent parameters on the skin friction coefficient and rates of heat and mass transfer in terms of the Nusselt number and Sherwood numbers are presented numerically in tabular form. The results shows that the observed parameters have significance influence on the flow, heat and mass transfer.
\end{abstract}

\section{Introduction}

Heat and mass transfer from different geometries embedded in porous media has many engineering and geophysical applications such as drying of porous solids, thermal insulations, cooling of nuclear reactors, crude oil extraction, underground energy transport, e.t.c. Micropolar fluids are those consisting of randomly oriented particles suspended in a viscous medium, which can undergo a rotation that can affect the hydrodynamics of the flow, making it a distinctly non- Newtonian fluid. They constitute an important branch of non-Newtonian fluid dynamics where microrotation effects as well as microinertia are exhibited. The theory of Micropolar fluids originally developed by Eringen[1] has been a popular field of research in recent years. Eringen's theory has provided a good model for studying a number of complicated fluids, such as colloidal fluids, polymeric fluids and blood. Micropolar fluid flow induced by the simultaneous action of buoyancy forces is of great interest in nature and in many industrial applications as drying processes, solidification of binary alloy as well as in astrophysics, geophysics and oceanography. 
When the strength of the magnetic field is strong, one cannot neglect the effect of Hall current. It is of considerable importance and interest to study how the results of the hydrodynamical problems get modified by the effect of Hall currents. Hall currents give rise to a cross flow making the flow three dimensional. Several authors [2 -12] studied MHD flow of a micropolar fluid. Rakesh[13] studied effect of slip conditions and Hall current on unsteady MHD flow of a viscoelastic fluid past an infinite vertical porous plate through porous medium.

We extended the work of Rakesh [13] by incorporating angular momentum and concentration equations to study Hall current and slip conditions on heat and mass transfer of unsteady MHD flow of a viscoelastic micropolar fluid through a porous medium. The governing equations are solved analytically using perturbation method and effect of various physical parameters are discussed numerically and graphically.

\section{Mathematical Formulation}

We consider the unsteady flow of a viscous incompressible and electrically conducting viscoelastic micropolar fluid over an infinite vertical porous plate, subjected to a constant transverse magnetic field BO in the presence of thermal and concentration buoyancy effects. The induced mag- netic field is assumed to be negligible compared to the applied magnetic field. The $x^{*}$ - axis is taken along the planar surface in the upward direction and the $\mathrm{y}^{*}$ - axis taken to be normal to it as shown in fig.1. Due to the infinite plane surface assumption, the flow variables are function of $\mathrm{y}^{*}$ and the $t^{*}$ only.The plate is subjected to a constant suction velocity $V_{0}$.

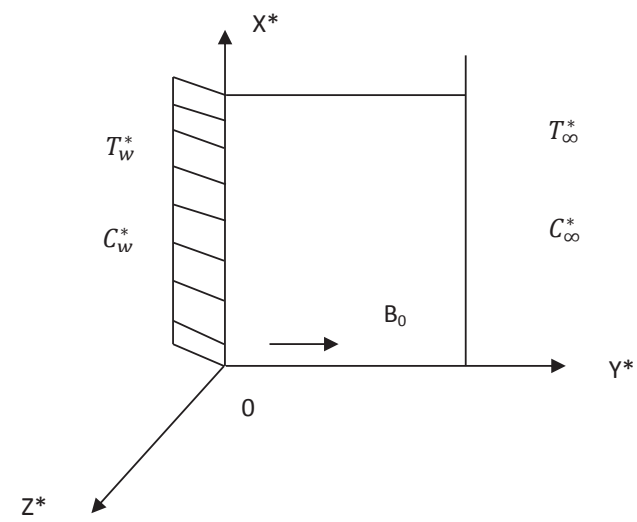

Fig. 1: Physical mode.

The governing equations of flow under the usual Boussinesq approximation are given by

$$
\frac{\partial v^{*}}{\partial y^{*}}=0
$$

$\frac{\partial T^{*}}{\partial t^{*}}+\nu^{*} \frac{\partial T^{*}}{\partial y^{*}}=\frac{k}{\rho C_{p}} \frac{\partial^{2} T^{*}}{\partial y^{* 2}}$$$
\frac{\partial C^{*}}{\partial t^{*}}+v^{*} \frac{\partial C^{*}}{\partial y^{*}}=D \frac{\partial^{2} C^{*}}{\partial y^{* 2}}
$$

The appropriate boundary conditions for the problem are

$$
\begin{aligned}
& u^{*}=L^{*}\left(\frac{\partial u^{*}}{\partial y^{*}}\right), w^{*}=L^{*}\left(\frac{\partial u^{*}}{\partial y^{*}}\right), N_{1}^{*}=-n \frac{\partial u^{*}}{\partial y^{*}}, N_{2}^{*}=n \frac{\partial w^{*}}{\partial y^{*}} T^{*}=T_{\infty}^{*}+\left(T_{w}^{*}-T_{\infty}^{*}\right) e^{i \omega^{*} t^{*}} \\
& C^{*}=C_{\infty}^{*}+\left(C_{w}^{*}-C_{\infty}^{*}\right) e^{i \omega^{*} t^{*}} \text { at } y^{*}=0 \\
& u^{*} \rightarrow 0, w^{*} \rightarrow 0, N_{1}^{*} \rightarrow 0, N_{2}^{*} \rightarrow 0 T^{*} \rightarrow T_{\infty}^{*}, C^{*} \rightarrow C_{\infty}^{*} \text { at } y^{*} \rightarrow \infty
\end{aligned}
$$


Where $u^{*}, v^{*}$ and $w^{*}$ are velocity components along $x^{*}, y^{*}$ and $z^{*}$-axis respectively,$N_{1}^{*}$ and $N_{2}{ }^{*}$ are microrotation components along $x^{*}$ and $z^{*}$-axis respectively, $v$ is the Kinematic viscosity, vris the Kinematic micro-rotation viscosity, $\mathrm{K}_{0}$ is the limiting viscosity, $g$ is the acceleration due to gravity, $\beta_{\mathrm{t}}$ and $\beta_{c}$ are the coefficients of thermal expansion and concentration expansion respectively, $T^{*}$ is the dimensional temperature of the fluid, $T_{w}{ }^{*}$ and $T_{\infty}{ }^{*}$ denotes the temperature at the plate and temperature far away from the plate respectively, $C^{*}$ is the dimensional concentration of the solute, $C_{w}{ }^{*}$ and $C_{\infty}{ }^{*}$ are concentration of the solute at the plate and concentration of the solute far from the plate respectively, $K^{*}$ is the permeability of the porous medium, $k$ is the thermal conductivity of the medium, $\rho$ is the density of the fluid, $j^{*}$ is the micro-inertia density or micro-inertia per unit mass, $y$ is the spin gradient viscosity, $L^{*}$ is the characteristic length, $\omega^{*}$ is the dimensional frequency of oscillation, $\sigma$ is the electrical conductivity, $m$ is the Hall current parameter and $D$ is the molecular diffusivity.

The constant that related to microgyration vector and shear stress is $n$. Further, $0 \leq n \leq 1$. The case $n=$ 0 represents concentrated particle flows in which the microelement close to the wall surface are unable to rotate. This case is also known as the strong concentration of microelements. The case $n=0.5$ indicates the vanishing of anti-symmetric part of the stress tensor and denotes weak concentration of microelements. The case $n=1$ is used for the modeling of turbulent boundary layer flows. We shall consider $n=0$ and $n=0.5$.

Let us introduce the following dimensionless variables:

$$
\begin{aligned}
& u=\frac{u^{*}}{V_{0}}, v=\frac{v^{*}}{V_{0}}, w=\frac{w^{*}}{V_{0}} \eta=\frac{V_{0} y^{*}}{\nu}, \\
& N_{1}=\frac{\nu N_{1}^{*}}{V_{0}^{2}}, N_{2}=\frac{\nu N_{2}^{*}}{V_{0}^{2}} t=\frac{t^{*} V_{0}^{2}}{4 \nu}, \\
& \omega=\frac{4 \nu \omega^{*}}{V_{0}^{2}}, h=\frac{V_{0} L^{*}}{\nu}, \\
& \theta=\frac{T^{*}-T_{\infty}^{*}}{T_{w}^{*}-T_{\infty}^{*}}, C=\frac{C^{*}-C_{\infty}^{*}}{C_{w}^{*}-C_{\infty}^{*}}, \quad J=\frac{V_{0}^{2} J^{*}}{\nu^{2}}
\end{aligned}
$$

Substituting equation (9) into equations (2) - (8) yield the following dimensionless equations:

$$
\begin{aligned}
& \frac{1}{4} \frac{\partial u}{\partial t}-\frac{\partial u}{\partial \eta}=(1+\beta) \frac{\partial^{2} u}{\partial \eta^{2}}-a \frac{\partial^{3} u}{\partial t \partial \eta^{2}}+\beta \frac{\partial N_{1}}{\partial \eta}-\frac{M}{1+m^{2}}(m w+u)+G r \theta+G c C-\frac{u}{K} \\
& \frac{1}{4} \frac{\partial w}{\partial t}-\frac{\partial w}{\partial \eta}=(1+\beta) \frac{\partial^{2} w}{\partial \eta^{2}}-a \frac{\partial^{3} w}{\partial t \partial \eta^{2}}-\beta \frac{\partial N_{2}}{\partial \eta}-\frac{M}{1+m^{2}}(w-m u)-\frac{u}{K}
\end{aligned}
$$

$\frac{1}{4} \frac{\partial N_{1}}{\partial t}-\frac{\partial N_{1}}{\partial \eta}=L \frac{\partial^{2} N_{1}}{\partial \eta^{2}}$

$\frac{1}{4} \frac{\partial N_{2}}{\partial t}-\frac{\partial N_{2}}{\partial \eta}=L \frac{\partial^{2} N_{2}}{\partial \eta^{2}}$

$\frac{1}{4} \frac{\partial \theta}{\partial t}-\frac{\partial \theta}{\partial \eta}=\frac{1}{\operatorname{Pr}} \frac{\partial^{2} \theta}{\partial \eta^{2}}$

$\frac{1}{4} \frac{\partial C}{\partial t}-\frac{\partial C}{\partial \eta}=\frac{1}{S c} \frac{\partial^{2} C}{\partial \eta^{2}}$

Where $\beta=\frac{\nu_{r}}{\nu}$ is the dimensionless viscosity ratio, $a=\frac{K_{0} V_{0}^{2}}{4 \nu^{2}}$ is the viscoelastic parameter, $M=\frac{\sigma B_{0}^{2} \nu}{\rho V_{0}^{2}}$ is the magnetic field parameter, $G r=\frac{\nu \beta_{i} g\left(T_{w}^{*}-T_{x}^{*}\right)}{\left.V_{s}^{s}\right)}$ is the Grashof number, $G c=\frac{\nu \beta_{c} g\left(C_{w}^{*}-C_{x}^{*}\right)}{V_{0}^{3}}$ is the modified
Grashof number, $P r=\frac{\nu \rho C_{p}}{K}$ is the Prandtl number, $S c=\frac{\nu}{D}$ is the Schmidt number, $K=\frac{K^{*} V_{0}^{2}}{\nu^{2}}$ is the permeability of the porous medium parameter and $L=\frac{\gamma V_{0}^{2}}{\partial \nu^{2} j}$ is the material parameter.

Also the boundary conditions becomes

$$
\begin{aligned}
& u=h \frac{\partial u}{\partial \eta}, w=h \frac{\partial w}{\partial \eta}, \theta=e^{i \omega t}, C=e^{i \omega t} \\
& N_{1}=-n \frac{\partial u}{\partial \eta}, N_{2}=n \frac{\partial w}{\partial \eta} \text { at } y=0 \\
& u \rightarrow 0, w \rightarrow 0, \theta \rightarrow 0, C \rightarrow 0 \\
& N_{1} \rightarrow 0, N_{2} \rightarrow 0 \text { at } y \rightarrow \infty
\end{aligned}
$$

We now simplified (10) - (13) by introducing $q=u+i w$ and $p=N_{1}+i N_{2}$ to have

$$
\frac{1}{4} \frac{\partial q}{\partial t}-\frac{\partial q}{\partial \eta}=(1+\beta) \frac{\partial^{2} q}{\partial \eta^{2}}-a \frac{\partial^{3} q}{\partial t \partial \eta^{2}}+i \beta \frac{\partial p}{\partial \eta}-\frac{M}{1+m^{2}}(1-i m) q+G r \theta+G c C-\frac{q}{K}
$$




$$
\begin{aligned}
& \frac{1}{4} \frac{\partial p}{\partial t}-\frac{\partial p}{\partial \eta}=L \frac{\partial^{2} p}{\partial \eta^{2}} \\
& \frac{1}{4} \frac{\partial \theta}{\partial t}-\frac{\partial \theta}{\partial \eta}=\frac{1}{P r} \frac{\partial^{2} \theta}{\partial \eta^{2}} \\
& \frac{1}{4} \frac{\partial C}{\partial t}-\frac{\partial C}{\partial \eta}=\frac{1}{S c} \frac{\partial^{2} C}{\partial \eta^{2}}
\end{aligned}
$$

And the corresponding boundary conditions are

$q=h \frac{\partial q}{\partial \eta}, \theta=e^{i \omega t}, C=e^{i \omega t}, P=\operatorname{in} \frac{\partial q}{\partial \eta}$,

at $y=0$

$q \rightarrow 0, \theta \rightarrow 0, C \rightarrow 0, P \rightarrow 0$

at $y \rightarrow \infty$

\section{Method of Solution}

In order to solve equations (17) - (20) subject to the boundary conditions (21), we assume a perturbation of the form:

$$
\begin{aligned}
& q=q_{0}(\eta) e^{i \omega t} \\
& P=P_{0}(\eta) e^{i \omega t} \\
& \theta=\theta_{0}(\eta) e^{i \omega t} \\
& C=C_{0}(\eta) e^{i \omega t}
\end{aligned}
$$

Substituting equation (22) into equations (17) (20), we obtain the following set of equations:

$\left(a_{1}-i a_{2}\right) q_{0}^{\prime \prime}+q_{0}^{\prime}-\left(a_{3}+i a_{4}\right) q_{0}=$

$=-G r \theta_{0}-G c C_{0}-i \beta P_{0}^{\prime}$

$L P_{0}^{\prime \prime}+P_{0}^{\prime}-\frac{i \omega}{4} P_{0}=0$

$\theta_{0}+\operatorname{Pr} \theta_{0}-\frac{i \omega \operatorname{Pr}}{4} \theta_{0}=0$

$C_{0}^{\prime \prime}+S c C_{0}^{\prime}-\frac{i \omega S c}{4} C_{0}=0$

Where $a_{1}=1+\beta, a_{2}=a \omega, a_{3}=\frac{M}{1+m^{2}}+\frac{1}{K}, a_{4}=\frac{\omega}{4}-\frac{M m}{1+m^{2}}$

The corresponding boundary conditions can be written as

$$
q_{0}=h \frac{\partial q_{0}}{\partial \eta}, \quad \theta_{0}=1, C_{0}=1, P_{0}=i n \frac{\partial q_{0}}{\partial \eta}
$$

$$
\begin{aligned}
& \text { at } y=0 \\
& q_{0}=0, \quad \theta_{0}=0, C_{0}=0, P_{0}=0 \\
& \text { at } y \rightarrow \infty
\end{aligned}
$$

The solution of (23) - (26) satisfying the boundary conditions (27) are given by

$$
\begin{aligned}
& q=\left(A_{1} e^{-r_{4} \eta}+A_{2} e^{-r_{2} \eta}+A_{3} e^{-r_{3} \eta}+A_{4} e^{-n \eta}\right) e^{i \omega t} \\
& P=B_{1} e^{i \omega t-n \eta} \\
& \theta=e^{i_{\omega} t-r_{2} \eta} \\
& C=e^{i \omega t-r_{3} \eta}
\end{aligned}
$$

Where

$$
\begin{aligned}
& a_{1}=1+\beta \\
& a_{2}=a \omega \\
& a_{3}=\frac{M}{1+m^{2}}+\frac{1}{K} \\
& a_{4}=\frac{\omega}{4}-\frac{M m}{1+m^{2}} \\
& r_{1}=\frac{1+\sqrt{1+i \omega L}}{2 L} \\
& r_{2}=\frac{P r+\sqrt{P r^{2}+i \omega P r}}{2} \\
& r_{3}=\frac{S c+\sqrt{S c^{2}+i \omega S c}}{2}
\end{aligned}
$$

$T_{1}=a_{1}-i a_{2}$

$T_{2}=a_{3}+i a_{4}$

$r_{4}=\frac{1+\sqrt{1+4 T_{1} T_{2}}}{2 T_{1}}$

$$
A_{2}=\frac{-G r}{r_{2}^{2} T_{1}-r_{2}-T_{2}}
$$$$
A_{3}=\frac{-G c}{r_{3}^{2} T_{1}-r_{3}-T_{2}}
$$$$
A_{1}=\frac{-h\left(r_{2} A_{2}+r_{3} A_{3}+r_{1} A_{4}\right)-\left(A_{2}+A_{3}+A_{4}\right)}{\left(1+h r_{4}\right)}
$$$$
B_{1}=-n i\left(r_{4} A_{1}+r_{2} A_{2}+r_{3} A_{3}+r_{1} A_{4}\right)
$$

$A_{4}=\frac{-r_{1} \beta n\left[r_{4} h r_{2} A_{2}+r_{4} h r_{3} A_{3}+r_{4} A_{2}+r_{4} A_{3}-\left(1+h r_{4}\right)\left(r_{2} A_{2}+r_{3} A_{3}\right)\right]}{\left(r_{1}^{2} T_{1}-r_{1}-T_{2}\right)\left(1+h r_{4}\right)-\beta n\left(r_{1}^{2} r_{4} h+n r_{4}+\left(1+h r_{4}\right) r_{1}^{2}\right)}$

The results are presented as velocity, microrotation, temperature and concentration profiles in figures $2-21$ below 


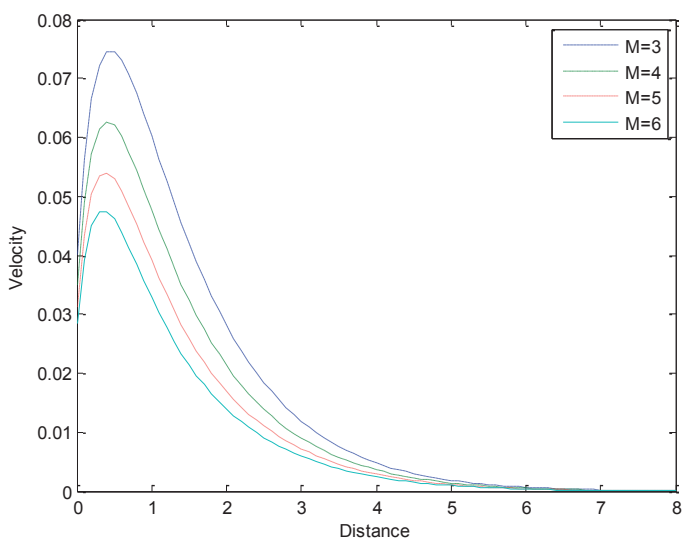

Fig. 2: Translational velocity profiles with different values of Magnetic field parameter.

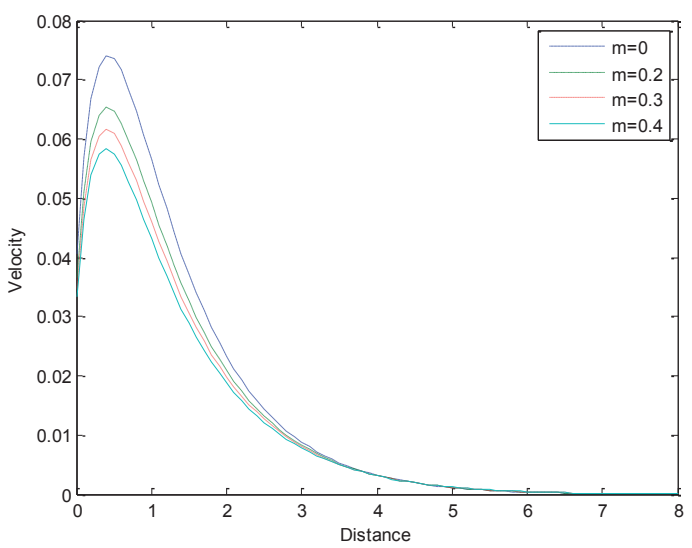

Fig. 3: Translational velocity profiles with different values of Hall current parameter.

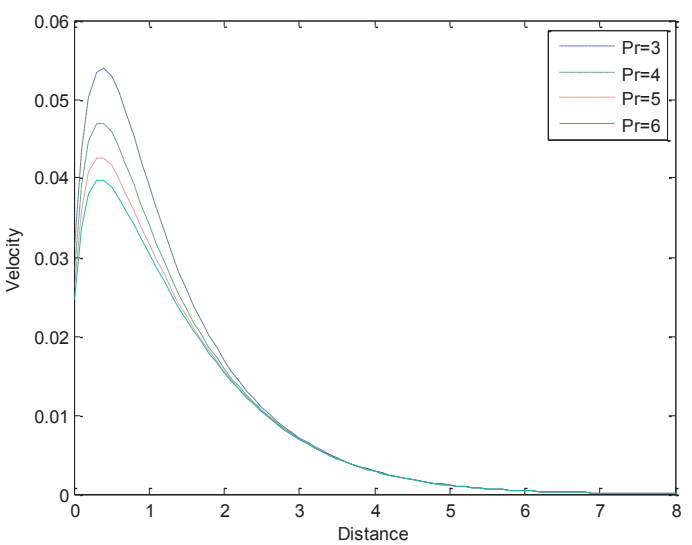

Fig. 4: Translational velocity profiles with different values Prandtl number.

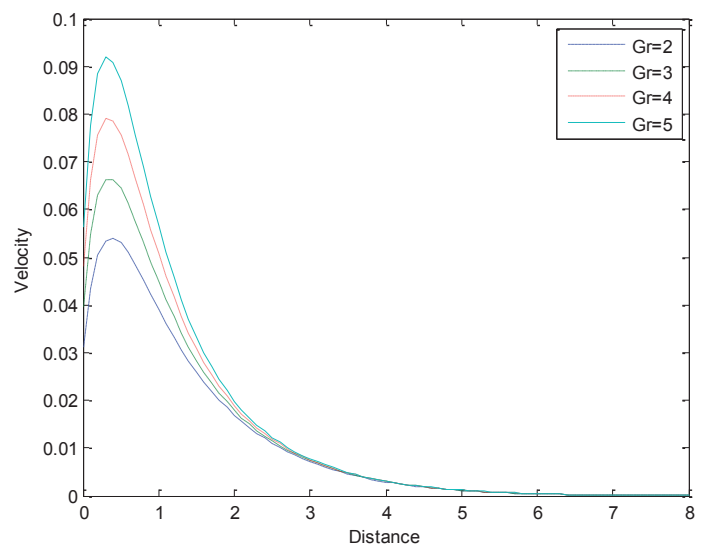

Fig. 5: Translational velocity profiles with different values of Grashof number.

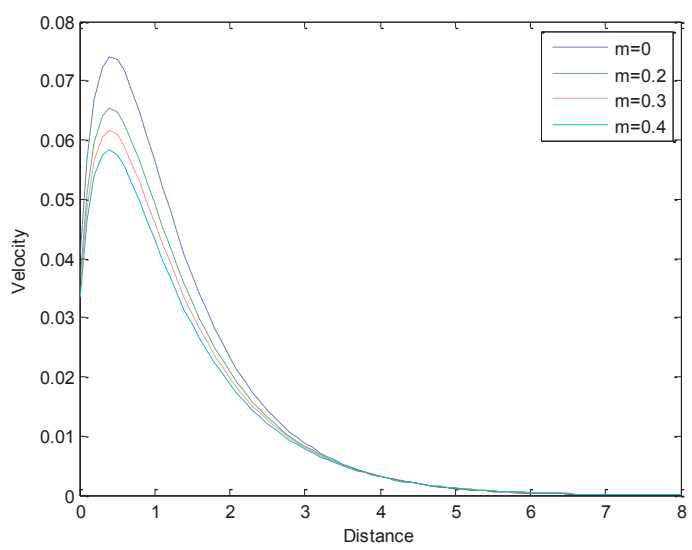

Fig. 6: Translational velocity profiles with different values of modified Grashof number.

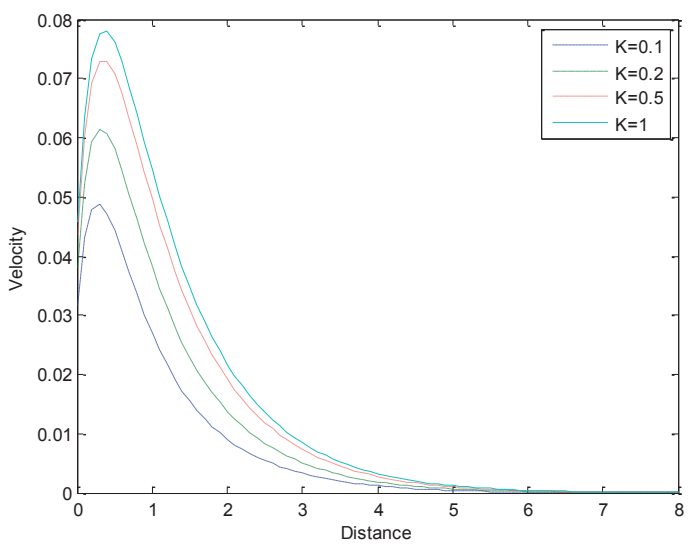

Fig. 7: Translational velocity profiles with different values of permeability of porous medium parameter. 


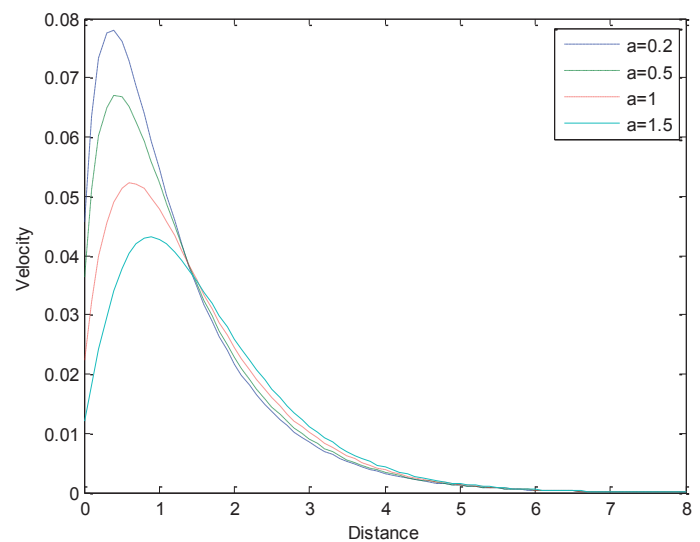

Fig. 8: Translational velocity profiles with different values of viscoelatic parameter.

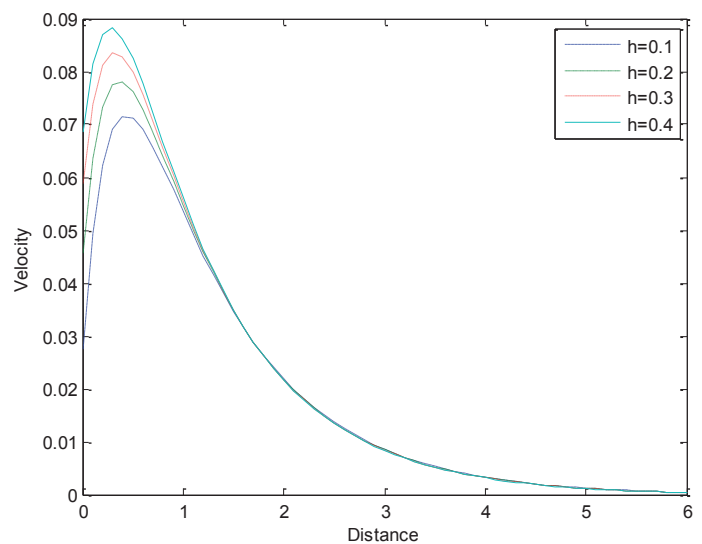

Fig. 9: Translational profiles velocity with different values of slip parameter.

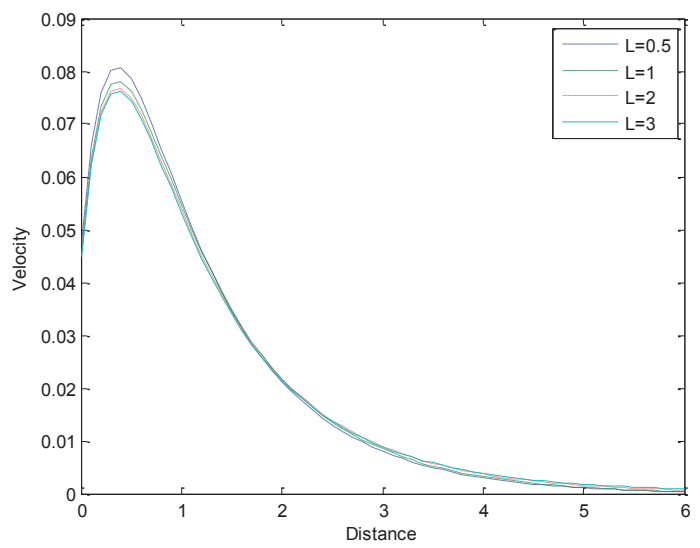

Fig. 10: Translational velocity profiles with different values of material parameter.

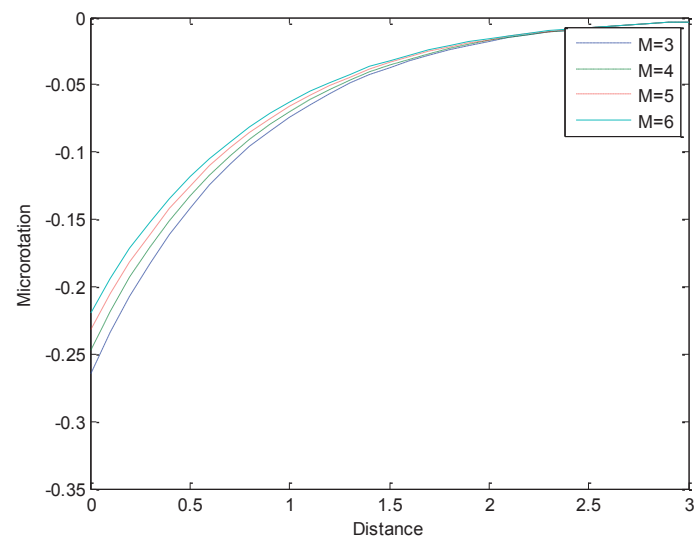

Fig. 11 a: Microrotational velocity profiles with different values of magnetic parameter.

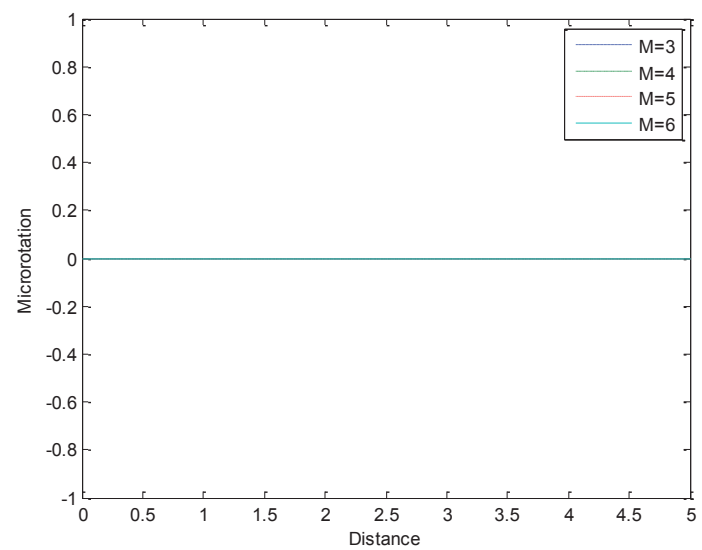

Fig. 11b: Microrotational velocity profiles with different values of magnetic parameter when $n=0$.

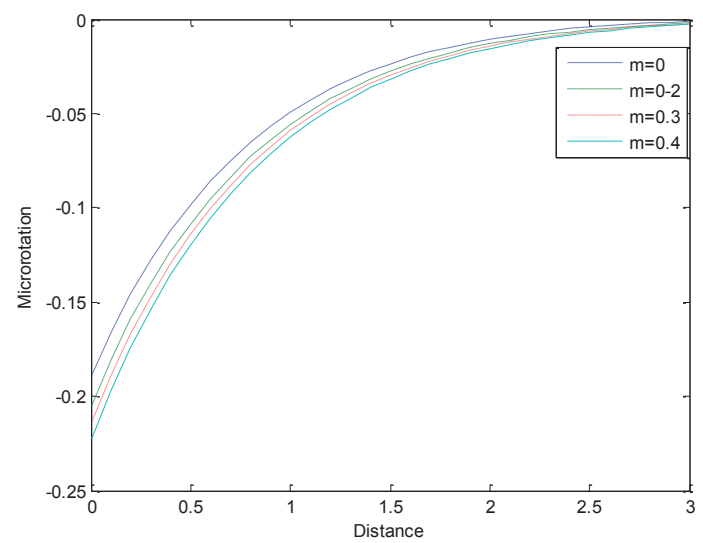

Fig. 12: Microrotational velocity profiles with different values of Hall current parameter. 


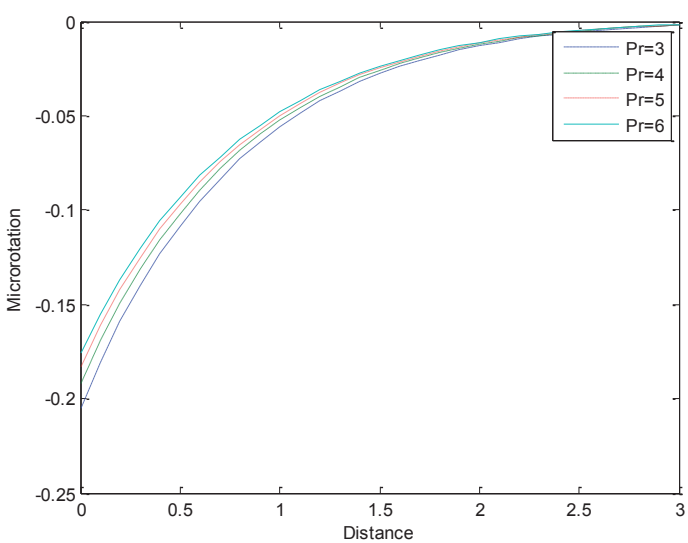

Fig. 13 Microrotational velocity profiles with different values of Prandt/ number.

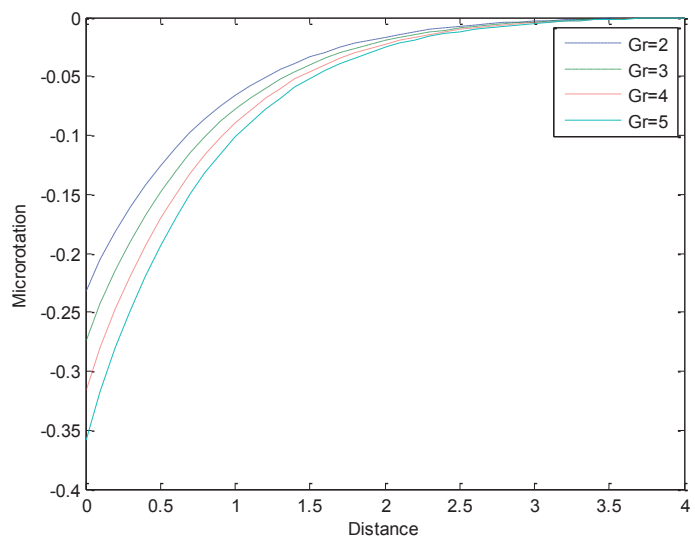

Fig. 14: Microrotational velocity profiles with different values of Grashof number.

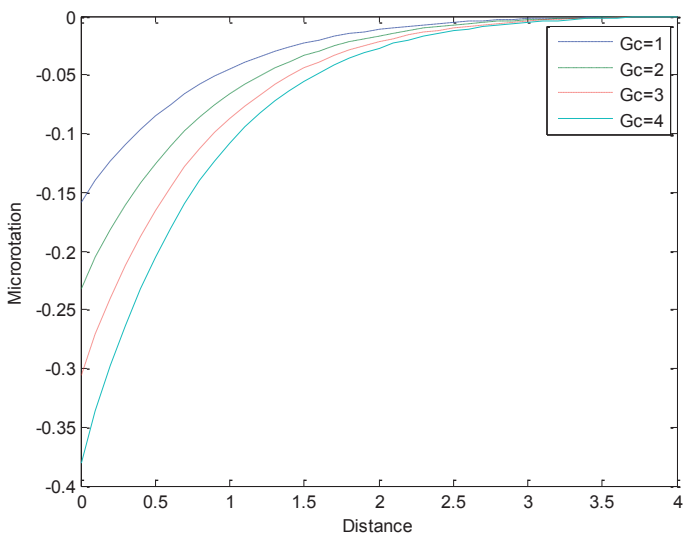

Fig. 15: Microrotational velocity profiles with different values of modified Grashof number.

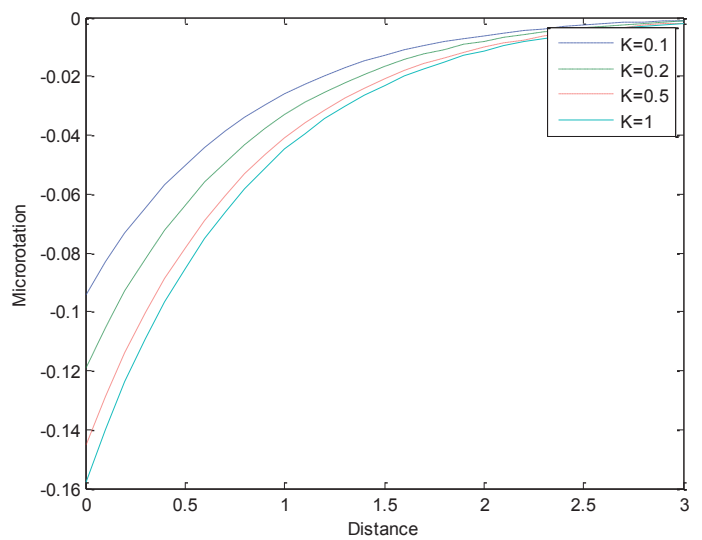

Fig. 16: Microrotational velocity profiles with different values of permeability of the porous medium.

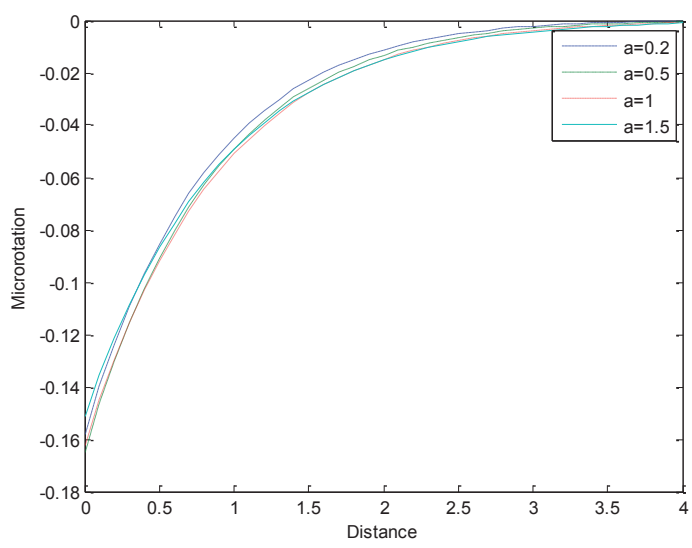

Fig. 17: Microrotational velocity profiles with different values of viscoelastic parameter.

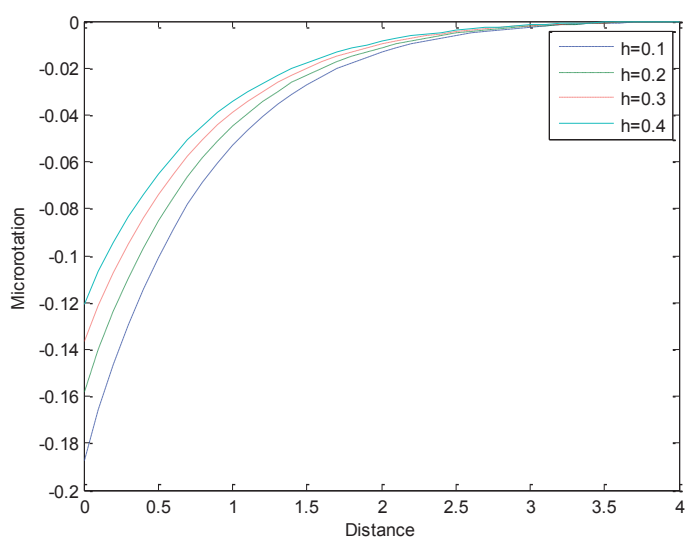

Fig. 18: Microrotational velocity profiles with different values of slip parameter. 


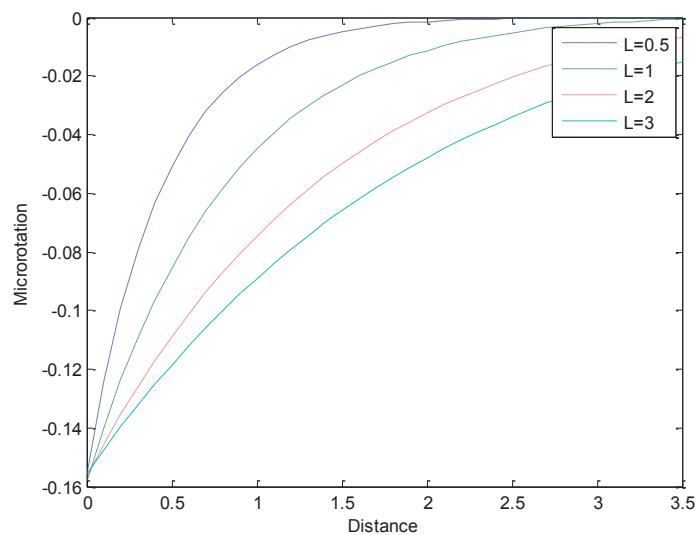

Fig. 19: Microrotational velocity profiles with different values of material parameter.

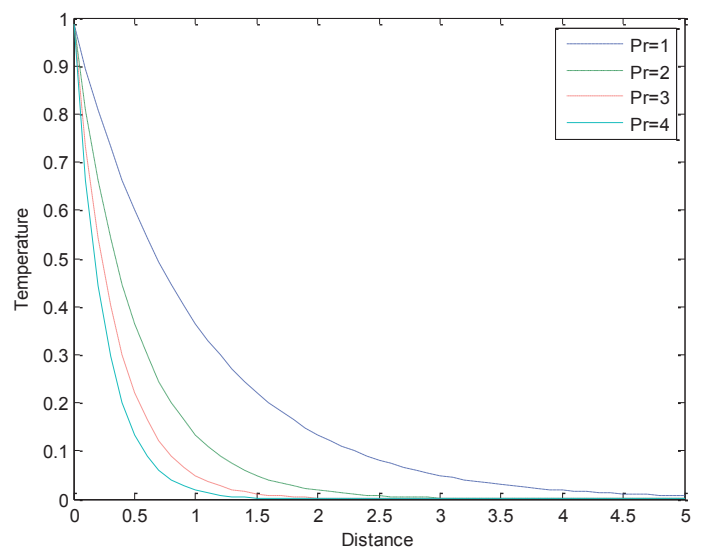

Fig. 20: Temperature profiles with different values of Prandt/ number.

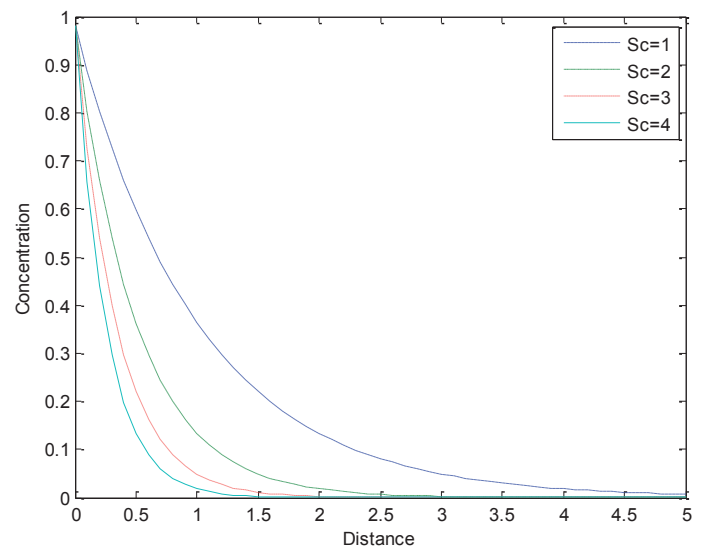

Fig. 21: Concentration profiles with different values of Schmidth number.
The local skin friction coefficient, couple stress coefficient, Nusselt number and Sherwood number are important physical quantities of engineering interest. The skin friction coefficient (Cf) at the wall is given by $C f=\frac{\tau_{w}}{\rho V_{0}^{2}}=[1+(1-n) L] q^{\prime}(0)$.

Where $\tau_{w}^{*}$ is the skin friction

$=-[1+(1-n) L]\left[r_{4} A_{1}+r_{2} A_{2}+r_{3} A_{3}+r_{1} A_{4}\right] e^{i \omega t}$

The couple stress coefficient $\left(C_{w}^{\prime}\right)$ at the plate is written as $C_{w}=\frac{M_{w} v^{2}}{V_{0}^{3}}=p^{\prime}(0)$ where Mw is the wall couple stress.

$$
\left.=-r_{1} B_{1} e^{i \omega t}\right)
$$

The rate of heat transfer at the surface in terms of the Nusselt number is given by

$$
\begin{gathered}
x u=\frac{x\left(\frac{\partial T^{*}}{\partial y^{*}}\right)}{\left(T_{\infty}^{*}-T_{w}^{*}\right)} \\
N u \operatorname{Re}_{x}^{-1}=-\theta(0)=r_{2} e^{i \omega t} \\
\text { Where } \operatorname{Re}_{x}=\frac{x V_{0}}{\nu}
\end{gathered}
$$

The rate of mass transfer at the surface in terms of the local Sherwood number is given by

$$
\begin{gathered}
S h=\frac{\left(\frac{\partial C^{*}}{\partial y^{*}}\right)_{y^{*}=0}}{C_{\infty}^{*}-C_{w}^{*}} \\
\operatorname{ShRe}_{x}^{-1}=-C^{\prime}(0)=r_{3} e^{i \omega t}
\end{gathered}
$$

The numerical result for skin friction coefficient, couple stress coefficient, Nusselt number and Sherwood number are shown in tables 1 - 3 below:

Table 1: Effect of $n, a, m$ and $h$ parameter on $C_{f}$ and $C_{w}{ }_{w}$ with $K=1$, $\omega t=\pi / 4, \beta=0.5, G r=2, G c=1, M=5, \operatorname{Pr}=3, L=1$.

\begin{tabular}{|l|l|l|l|l|l|}
\hline $\mathbf{N}$ & $\mathbf{a}$ & $\mathbf{m}$ & $\mathbf{h}$ & $\mathbf{C}_{\mathbf{f}}$ & $\mathbf{C}_{\mathbf{w}}$ \\
\hline 0 & 0.2 & 0.2 & 0.2 & 0.4728 & 0 \\
\hline 0.5 & 0.2 & 0.2 & 0.2 & 0.3651 & 0.1753 \\
\hline 1 & 0.2 & 0.2 & 0.2 & 0.2500 & 0.3613 \\
\hline 0.5 & 0.5 & 0.2 & 0.2 & 0.3023 & 0.1800 \\
\hline 0.5 & 1 & 0.2 & 0.2 & 0.2021 & 0.1747 \\
\hline
\end{tabular}




\begin{tabular}{|l|l|l|l|l|l|}
\hline 0.5 & 1.5 & 0.2 & 0.2 & 0.1246 & 0.1606 \\
\hline 0.5 & 0.2 & 0 & 0.2 & 0.3883 & 0.1661 \\
\hline 0.5 & 0.2 & 0.3 & 0.2 & 0.3560 & 0.1805 \\
\hline 0.5 & 0.2 & 0.4 & 0.2 & 0.3488 & 0.1861 \\
\hline 0.5 & 0.2 & 0.2 & 0.1 & 0.4344 & 0.2102 \\
\hline 0.5 & 0.2 & 0.2 & 0.3 & 0.3149 & 0.1503 \\
\hline 0.5 & 0.2 & 0.2 & 0.4 & 0.2767 & 0.1315 \\
\hline
\end{tabular}

Table 2: Effect $\operatorname{Pr}$ and $\omega$ parameter on $N u R e_{x}^{-1}$ with $n=0.5, K=1$, $\beta=0.5, G r=2, G c=1, M=5, \operatorname{Pr}=3, \omega t=\pi / 4, L=1, m=0.2, h=0.2, a=0.2$

\begin{tabular}{|l|l|l|}
\hline $\mathbf{P r}$ & $\boldsymbol{\omega}$ & $\mathrm{NuRe}_{x}^{-1}$ \\
\hline 3 & 1 & 1.9611 \\
\hline 4 & 1 & 2.6638 \\
\hline 5 & 1 & 3.3684 \\
\hline 3 & 0.2 & 0.5912 \\
\hline 3 & 0.5 & 1.3644 \\
\hline 3 & 1 & 1.9611 \\
\hline
\end{tabular}

Table 3: Effect of Sc and w parameter on $\operatorname{ShRe}_{x}^{-1}$ with $n=0.5$, $m=0.2, h=0.2, \beta=0.5, G r=2, G c=1, M=5, \operatorname{Pr}=3$

\begin{tabular}{|l|l|l|}
\hline \multicolumn{1}{|c|}{ Sc } & $\boldsymbol{\omega}$ & $\operatorname{ShRe}_{x}^{-1}$ \\
\hline 2 & 1 & \\
\hline 3 & 1 & 1.9611 \\
\hline 4 & 1 & 2.6638 \\
\hline 1 & 0.2 & 0.1965 \\
\hline 1 & 0.5 & 0.4454 \\
\hline 1 & 1 & 0.5811 \\
\hline
\end{tabular}

\section{Discussion}

Unsteady incompressible and electrically threedimensional flow of a viscoelastic micropolar fluid over an infinite vertical porous plate through porous medium was studied. Numerical evaluation of the analytical solutions reported in the previous section was performed and the results are presented in graphical and tabular form. This was done to illustrate the influence of the various parameter involved. In this study, we have chosen $\omega t=\pi / 4$ while other parameters are varied over a range.

The effect of magnetic field parameter on velocity distribution profiles across the boundary layer are presented in Fig. 2. It is obvious that the effect of increasing values of the magnetic field parameter $M$ results in a decreasing velocity distribution across the boundary layer. This is due to the fact that the effect of a transverse magnetic field give rise to a resistive type force called the Lorentz force. The force has the tendency to slow the motion of the fluid. Fig.3 display the effect of Hall current parameter on the translational velocity distribution profiles. It is noticed that the Hall current parameter decreases the velocity. Fig. 4 present the translational velocity distribution profiles for different values of the Prandtl number(Pr). The results show that the effect of increasing values of the pranddtl number results in a decrease in the velocity. Fig. 5 and Fig. 6 illustrates the velocity profiles for different values of Grashof number (Gr) and modified Grashof number $(\mathrm{Gc})$ respectively. It can be seen that an increase in $\mathrm{Gr}$ or Gc leads to a rise in velocity profiles. Fig.7 depict the effect of permeability of the porous medium parameter(K) on velocity distribution profiles and it is obvious that as permeability parameter(K) increases, the velocity increases along the boundary layer thickness which is expected since when the holes of porous medium become larger, the resistive of the medium may be neglected. Fig. 8 shows the influence of the viscoelastic parameter on translational velocity profiles. The velocity decreases as viscoelastic parameter increases in the vicinity of the plate but the reverse happens as one moves away from it. Fig.9 illustrates the variation of slip parameter with translational distribution profiles. As the parameter increases the velocity increases. It is expected since the slip parameter has the tendency to reduce the friction forces which increases fluid velocity. Fig. 10 show the translational velocity profiles with different values of material parameter. And the effect of the material parameter is to decrease the translational velocity.

Fig.11a and Fig. $11 \mathrm{~b}$ depict the microrotational velocity profiles for different values of magnetic field parameter respectively. In contrast to translational velocity distribution profiles, the microrotational velocity distribution profiles increases with increase in the magnetic field parameter when $n=0.5$ while it remain constant when $\mathrm{n}=0$ as shown in Fig $11 \mathrm{~b}$. It is clear from these figures that microrotational effect are more pronounced for $n=0.5$ in comparison to when $n=0$. Fig. 12 illustrates the microrotational velocity distribution for different values of Hall current parameter. The figure shows that as Hall current parameter increases, microrotational velocity decreases. Fig. 13 shows that as Prandtl number increases, microrotational velocity increases. Fig. 14 and Fig. 15 
elucidate that the effect of increasing Gr or Gc is to decrease microrotational velocity. The effects due to permeability of the porous medium parameter(K) on microrotational velocity is shown in Fig. 16. It is observed that as the parameter increases, the microrotational velocity decreases. Fig. 17 illustrate the microrotational velocity distribution for different values of viscoelastic parameter(a). The figure shows that asviscoelastic parameter increases, the microrotational velocity decreases. Fig. 18 shows that the effect of increasing slip parameter is to increase the microrotational velocity. Fig. 19 illustrate the effect of material parameter $(\mathrm{L})$ on microrotational velocity profiles. The profiles decreases as the parameter increases. Fig. 20 presents the effect of the Prandtl number $\operatorname{Pr}$ on the temperature profiles. Increasing the value of $\operatorname{Pr}$ has the tendency to decrease the fluid temperature in the boundary layer as well as the thermal boundary layer thickness. This causes the wall slope of the temperature to decrease as Pr is increasing causing the Nusselt number to increase as can be clearly seen in table 2. Fig. 21 shows concentration distribution profiles for different values of Sc. It can be noted from the figure that the concentration of the fluid decreasesas the Sc increases

Table1 shows the effects of constant that related to microgyration vector and shear stress ( $n$ ), viscoelastic parameter(a), Hall current parameter(m) and slip parameter( h) on skin friction coefficient and couple stress coefficient. It is observed that increase in value of $n$ decreases skin friction coefficient which is not surprising since $n=0$ represents strong concentration and $n=0.5$ represents weak concentration of the microelements while couple stress coefficient increases with increase in $\mathrm{n}$. Increase in viscoelastic parameter(a) decreases both skin friction coefficient and couple stress coefficient, increase in Hall prarmeter $(m)$ decreases skin friction coefficient and increases couple stress coefficient, and increase in slip parameter(h)decreases both skin friction coefficient and couple stress coefficient. Table 2 show the effect of Prandtl number(Pr) and frequency of oscillation parameter $(\omega)$ on the Nusselt number. The Nusselt number increases as both parameter increases. This shows that the surface heat transfer from the porous plate increases with the increasing values of $\operatorname{Pr}$ and $\omega$. Table 3 shows that the effect of increasing the $\mathrm{Sc}$ and $\omega$ is to increase the rate of mass transfer. These results are in good agreement with Modather [11], Roslinda [12] and Rakesh [13].

\section{Conclusion}

An analytical study of the MHD heat and mass transfer flow of an incompressible, elecyrically conducting viscoelatic micropolar fluid over an infinite vertical porous plate in through porous medium was conducted. The results are discussed through graphs and tables for different values of parameters entering into the problem. Following conclusions can be drawn from the results obtain:

- In the presence of a uniform magnetic field, increases in the strength of the applied magnetic field decelerated the fluid motion along the wall of the plate inside the boundary layer, whereas the microrotational velocity of the fluid along the wall of the plate increased.

- Increase in Hall current parameter decreases the momentum and thermal boundary layer thickness while it decelerate microrotational velocity.

- The Nusselt number increased as the PrandtI number and oscillation frequency parameter increased.

- The Sherwood number increased as the Schmidt number and oscillation frequency parameter increased.

- The slip parameter decreases both skin friction coefficient and couple stress coefficient.

\section{References}

[1] Erigen A.C, Theory of micropolar fluids J. math.mech. 16 pp.1-18.

[2] Srinivasachanya.D., Ramreddy. Ch.,Soret and Dufour effect on mixed convection in a non-Darcy porous medium saturated with micropolar fluid, Non-analysis modelling and control, vol.16,No.1, 100-115,2011.

[3] Rehbi, A.D., Tariq, A.A. Benbella, A.S. Mahoud, A.A. Unsteady natural convection heat transfer of micropolar fluid over a vertical surface with constant Heat flux, Turkish J. Eng. Env. Sci. Vol. 31, pp. 225-233 (2007).

[4] Eldabe, N.T. and Ouat, M.E., Chebyshew finite difference method for heat and mass transfer in hydromagnetic flow of a micropolar fluid past a stretching surface with Ohmic heating and viscous dissipation, Appl. Math. Comput., 177, pp. 561-571, 2006.

[5] Keelson, N.A., Desseaux, A. Effects of surface condition on flow of a micropolar fluid driven by a porous stretching sheet, Int. J. Eng. Sci, 39, pp. 1881-1897, 2001.

[6] Sunil, A. Sharma, A. Bharti, P.K. and Shandi, R.G. Effect of rotation on a layer of micropolar ferromagnetic fluid heated from below saturating a porous medium, International Journal of Engineering science, vol. 44 no. 11-12, pp. 683- 
698, 2006.

[7] Mahmoud, M.A.A., Thermal radiation effects on MHD flow of a micropolar fluid over a stretching surface with variable thermal conductivity, Physical A. 375, pp. 401-410, 2007

[8] Magdy, A.C., Free convection flow of conducting micropolar fluid with thermal relaxation including heat sources, Journal of Applied Mathematics, Vol. 2, 70.4 pp.271-292,2005.

[9] Patil, P.M. and Kulkarni P.S. Effects of chemical reaction on free convective flow of a polar fluid through a porous medium in the presence of internal heat generation, Int.Therm. Sci. 4. Pp. 1043-1054,2008.

[10] Chaudhary, R.C. and Abhay K.J. Effect of chemical reaction on MHD micropolar fluid flow past a vertical plate in slipflow regime. Appl. Math. Mech. Engl. Ed. 29(9): 117-1194, 2008.

[11] Modather M, Rashad A.M, Chamkha A.J. Study of MHD heat and mass transfer oscillatory flow of a micropolar fluid over a vertical permeable plate in a porous medium. Turkis J. । Zeng. Env. Sci. vol33, 245-257, 2009.

[12] Roslinda ,N.and Anuar, I. loan, P. Unsteady boundary layer flow over a stretching sheet in a micropolar fluid. International journal of engineering and applied science 4:7,2008.

[13] Rakesh, K.and Khem, C. Effect of slip conditions and Hall current on unsteady MHD flow of a viscoelastic fluid past an infinite vertical porous plate through porous medium. International journal of engineering sciences and technology Vol.3 No.4,0975-5462,2011. 